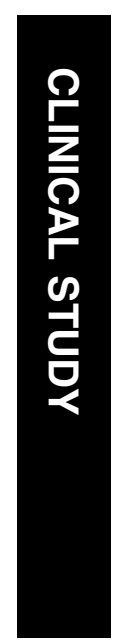

\title{
Volumetric colour Doppler imaging: a useful tool for the determination of ocular blood flow in glaucoma patients?
}

\begin{abstract}
Purpose Disturbed ocular haemodynamics are discussed to contribute to the pathogenesis of glaucoma. Up to now there is no method available allowing direct determination of blood flow, which is the most relevant dimension for studies on haemodynamics. In this study, volumetric colour Doppler imaging (vCDI) is evaluated systematically in glaucoma patients.
\end{abstract}

Methods A Siemens Elegra ultrasound set-up with a linear $7.5 \mathrm{MHz}$ probe was used for all CDI measurements. For vCDI, the crosssectional area of a vessel and the flow velocity is
O Zeitz'1,3, SE Vilchez ${ }^{1,2,3}$, ET Matthiessen', G Richard ${ }^{1}$ and M Klemm ${ }^{1}$

\section{Introduction}

Various ocular diseases are associated with alterations in ocular blood flow. This includes not only occlusion of ocular arteries and veins but also very common and widespread diseases like glaucoma, age-related macular degeneration and diabetic retinopathy. Disturbances of ocular blood flow in glaucoma patients are subject of intensive research and have also been postulated as an important therapeutic target. ${ }^{1,2}$ Thus, there is a strong need for reliable procedures and instruments for clinical evaluation of ocular perfusion in these patients.

Recent advances in the field of noninvasive technologies have led to substantial improvement in the clinical evaluation of ocular haemodynamics. At present, there are predominantly four basic methods to assess ocular perfusion: the evaluation of the ocular pulse amplitude (OPA) by the method of Langham, ${ }^{3,4}$ scanning laser Doppler flowmetric techniques like the Heidelberg Retina Flowmeter, ${ }^{5}$ fluorescence angiographic procedures, ${ }^{6,7}$ and colour Doppler imaging (CDI). ${ }^{8}$ Despite technical progress, none of the available techniques mentioned above allows one to measure ocular perfusion directly and quantitatively. All methods remain estimative. None of the methods except fluorescence angiography is able to determine blood flow as volume per time in a certain vessel, and even fluorescence angiography allows this only in funduscopic visible vessels ${ }^{7}$ but not in the orbit where the ciliary arteries are located that supply the optic nerve head with blood.

A more recent approach to determine ocular blood flow is to perform volumetric measurements with CDI set-ups. During this procedure, mean blood flow velocity and \footnotetext{
determined. From both these parameters blood flow can be calculated. Ocular pulse amplitude (OPA) was assessed by the method of Langham using a pneumatic applanation tonometer. Results (1) Velocity measurements using CDI in the ophthalmic artery and central retinal artery were highly reproducible $(n=20)$. In contrast, reproducibility of vCDI measurements was low $(n=20)$. Reproducibility improved if five vCDI measures were averaged. (2) Results from two different CDI-operators did not differ regarding the velocity measurements, but there was a difference in vCDI measurements $(n=20)$. (3) Results from vCDI did not correlate with measurements of OPA in 69 patients. (4) In 15 patients, vCDI failed to detect changes of ocular perfusion induced by the application of dorzolamide.

Conclusion vCDI is not applicable in ophthalmology at present.

Eye (2006) 20, 668-673. doi:10.1038/sj.eye.6701935; published online 3 June 2005

Keywords: colour Doppler imaging; glaucoma; dorzolamide

\author{
${ }^{3}$ Both these authors \\ contributed equally \\ ${ }^{1}$ Universitätsklinikum
Hamburg-Eppendorf, Klinik
und Poliklinik für
Augenheilkunde, Hamburg,
Germany
²Centro Oftalmológico del
Noroeste, Los Mochis,
México
Correspondence: O Zeitz,
Universitätsklinikum
Hamburg-Eppendorf,
Klinik und Poliklinik für
Augenheilkunde,
Martinistr. 52, Hamburg
D- 20246,
Germany
Tel: +49 040428032350 ;
Fax: +49 040428034906 .
E-mail: zeitz@
uke.uni-hamburg.de
Received: 12 November
2004
Accepted: 12 April 2005
Published online: 3 June
2005 \\ ${ }^{1}$ Universitätsklinikum
Hamburg-Eppendorf, Klinik
und Poliklinik für
Augenheilkunde, Hamburg,
Germany
²Centro Oftalmológico del
Noroeste, Los Mochis,
México
Correspondence: O Zeitz,
Universitätsklinikum
Hamburg-Eppendorf,
Klinik und Poliklinik für
Augenheilkunde,
Martinistr. 52, Hamburg
D- 20246,
Germany
Tel: +49 040428032350 ;
Fax: +49 040428034906 .
E-mail: zeitz@
uke.uni-hamburg.de
Received: 12 November
2004
Accepted: 12 April 2005
Published online: 3 June
2005 \\ ${ }^{1}$ Universitätsklinikum
Hamburg-Eppendorf, Klinik
und Poliklinik für
Augenheilkunde, Hamburg,
Germany
²Centro Oftalmológico del
Noroeste, Los Mochis,
México
Correspondence: O Zeitz,
Universitätsklinikum
Hamburg-Eppendorf,
Klinik und Poliklinik für
Augenheilkunde,
Martinistr. 52, Hamburg
D- 20246,
Germany
Tel: +49 040428032350 ;
Fax: +49 040428034906 .
E-mail: zeitz@
uke.uni-hamburg.de
Received: 12 November
2004
Accepted: 12 April 2005
Published online: 3 June
2005
}

1


diameter of the vessel is determined. Flow is then calculated by multiplication of these parameters. The method is termed as volumetric colour Doppler imaging (vCDI). vCDI is well established in different disciplines of medicine other than ophthalmology. For example, in cardiology, flow through the cardiac valves is assessed by a vCDI method in routine examination. ${ }^{9}$ In neurology, a vCDI procedure can be applied to determine blood flow in cranial vessels. A related procedure based on a commercial evaluation software provided by Advanced Technology Laboratories (ATL, a subsidiary of Phillips Medical Systems) was thought to be applicable, in previous work, at least for the ophthalmic artery (OA). ${ }^{10}$ This report of Orge and co-workers was followed by an intensive discussion of their results. Other groups doubted the reliability of the method, but, in summary, it was postulated that this approach might be not far away from a definitive solution to the problem of measuring ocular blood flow. ${ }^{11,12}$

This controversy led us to initiate a systematic evaluation of the vCDI method on a methodological and application, level with a modern, high-end ultrasound system to clarify whether vCDI and related methods are applicable in ophthalmology.

\section{Methods and patients}

The study was performed in accordance with institutional, national, and international guidelines and was approved by the local ethics committee.

\section{Study protocol}

Evaluation of vCDI by the present study was performed in four steps:

(1) Reproducibility of results was tested in 20 patients by performing five repeated measurements in the same individual.

(2) Results from the same patient but using two different CDI operators (SEV and OZ), were compared to determine investigator dependency of results.

(3) Results from vCDI were correlated with measurements of ocular blood flow by the Langham method based on assessment of the OPA in 69 patients.

(4) Finally, in 15 patients, the capacity of the method to identify changes of ocular perfusion was tested by topically applying dorzolamide, an inhibitor of the carbonic anhydrase, which has been shown to increase ocular perfusion in glaucoma patients. ${ }^{13-15}$

The study was performed under blinded conditions. Thus, evaluation of all four steps of the study was performed after completion of the entire data collection.

\section{Patients}

Adult patients who attended the clinic from June to November 2003 with a confirmed diagnosis of glaucoma were enrolled in this study. The patients underwent vCDI and LOBF measurement on the same day for correlation of methods. Patients who presented to the clinic with progressive normal tension glaucoma and received additional dorzolamide, underwent vCDI measurements of the one randomly chosen eye. Dorzolamide was applied three times a day in both eyes. vCDI was performed shortly before and after 3-5 weeks of treatment. Patients with an intraocular pressure higher than $21 \mathrm{mmHg}$ prior to therapy were not included in the dorzolamide branch of the study. In the dorzolamide group, systemic medication of the patients did not change between their first and second examination. Patients who had undergone ophthalmic surgery of the right eye, except cataract surgery, were not included into the study.

All examinations were performed with the patient sitting in an upright posture. All patients rested for several minutes before the examination started.

\section{Colour Doppler imaging}

CDI was performed with a Sonoline Elegra Advanced System (Siemens, Erlangen, Germany) using a phased array transducer type 7.5L40. (Siemens, Erlangen, Germany), as described previously. ${ }^{16}$ Ultrasound frequency was $7.5 \mathrm{MHz}$ in the pulsed Doppler mode. The transducer was carefully set on the closed eye lid without exerting pressure on the bulb. Acoustic coupling between transducer and skin was optimized by a carbomeric gel (Vidisic ${ }^{\circledR}$, Dr Mann Pharma, Germany). After identification of the optic nerve as a landmark, the following vessels were identified in the colour Doppler mode: central retinal artery (CRA), short and long posterior ciliary arteries (SPCA and LPCA) and OA. Flow velocity in the CRA was measured along its course through the optic nerve (Figure 1). Velocities in the SPCA and LPCA were recorded before entering the sclera. Flow velocity in the OA was measured close to its crossing of the optic nerve. Pulsed Doppler mode was used to measure flow velocities. The angle between transducer and orientation of the vessel was corrected. Gain and threshold were adjusted individually for each examination until noise disappeared, but then were kept constant during the entire procedure. Pulse repetition frequency was minimized to avoid aliasing (typically $5208 \mathrm{~Hz}$ for the OA, and $2500 \mathrm{~Hz}$ for the SPCA and LPCA in the Doppler mode). Sample size for all vessels was set constantly to $1.5 \mathrm{~mm}$. 


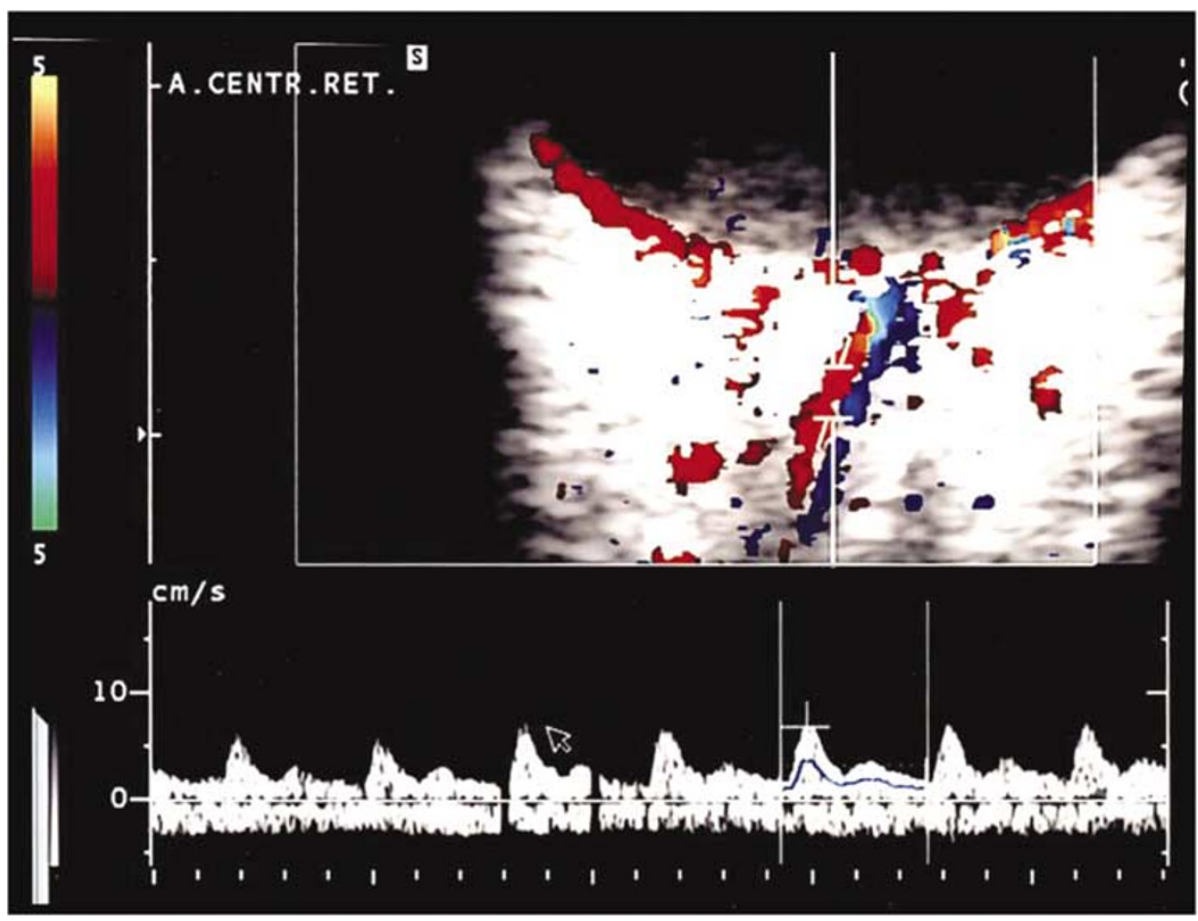

Figure 1 Original CDI-registration of the CRA.

The changes of blood flow velocities over the course of a cardiac cycle were recorded continuously. Peak systolic velocity and end diastolic velocity (EDV) can be determined directly, and time average mean velocity (TAMn), pulsatility index and resistive index are calculated automatically by the CDI software.

In addition to the basic CDI protocol, for vCDI measurements the diameter $(d)$ of CRA and OA was assessed in the colour Doppler mode (C-mode) at the same position where shortly before the velocity had been measured. Calculation of blood flow (volume per time; $\mathrm{d} V / \mathrm{d} t$ ) was performed by the formula $\mathrm{d} V /$ $\mathrm{d} t=\mathrm{TAMn} \times(0.5 \times d)^{2} \times \pi$. Dimension of $\mathrm{d} V / \mathrm{d} t$ was adjusted to $\mu \mathrm{l} / \mathrm{min}$.

\section{Langham-OBF measurements}

Langham-OBF (LOBF) was performed with an OBF Tonometer 3000 with a pneumatic probe and disposable probe tips adapted to a slitlamp. The tonometer was set on the anaesthetized cornea of the patient and IOP was recorded for several seconds at an acquisition frequency of $200 \mathrm{~Hz}$. From the actual IOP and the amplitude of the oscillations of IOP, ocular blood flow was estimated by mathematical algorithms of Langham and To'Mey. ${ }^{3}$

\section{Dorzolamide}

Dorzolamide was obtained in the form of Trusopt ${ }^{\circledR}$ from MSD-Chibret, Munich, Germany. Trusopt ${ }^{\circledR}$ contains
$22.26 \mathrm{mg}$ dorzolamide hydrochloride per $1 \mathrm{ml}$. Further ingredients of Trusopt ${ }^{\mathbb{R}}$ are hydroxyethylcellulose, D-mannitol, sodium citrate, sodium hydroxide, benzalkonium chloride and water as solvent.

\section{Statistics}

Statistical analysis of the data was carried out with SPSS 10.0. All data are given as mean \pm standard error of means (SEM). Student's $t$-test for paired data was used for evaluation of the effect of dorzolamide as well as for the comparison of the results from both investigators. Reproducibility of the vCDI measurements was statistically tested by determination of the single and average measure intraclass correlation coefficient (SMIC and AMIC). $P<0.05$ was considered to be significant.

\section{Results}

\section{Reproducibility of results}

Velocity measurements using CDI in the OA and in the CRA were highly reproducible $(n=20$, SMIC $>0.70$;

Table 1). Low reproducibility for measuring of diameter, and thus for flow, was found $(n=20 ; \mathrm{SMIC}<0.70$ for diameter and volume; Table 1$)$. Reproducibility improved if five consecutive measures performed by one observer were averaged (AMIC $>0.70$ for diameter and volume; Table 1). 
Table 1 Reproducibility (intraobserver variability) and observer dependency (interobserver variability) of vCDI measurements: SMIC and AMIC $>0.70$ reflects acceptable reproducibility (see text)

\begin{tabular}{|c|c|c|c|c|c|}
\hline & \multicolumn{2}{|c|}{ Operator 1} & \multicolumn{2}{|c|}{ Operator 2} & \multirow{2}{*}{$\frac{\text { Observer dependency }}{\mathrm{P}}$} \\
\hline & SMIC & AMIC & SMIC & AMIC & \\
\hline OA TAMn & 0.7188 & 0.9274 & 0.8325 & 0.9613 & 0.84 \\
\hline OA diameter & 0.6074 & 0.8855 & 0.5612 & 0.8647 & 0.0008 \\
\hline OA flow & 0.6674 & 0.8266 & 0.4997 & 0.6808 & 0.02 \\
\hline CRA TAMn & 0.7525 & 0.9383 & 0.8335 & 0.8974 & 0.45 \\
\hline CRA diameter & 0.2650 & 0.6432 & 0.6363 & 0.8974 & 0.01 \\
\hline CRA flow & 0.4717 & 0.8170 & 0.5268 & 0.8477 & 0.002 \\
\hline
\end{tabular}

$P<0.05$ indicates significant differences between the measures of both observes and thus a high interobserver variability.

$\mathrm{SMIC}$ and $\mathrm{AMIC}=$ single and average measure intraclass correlation coefficient; $\mathrm{OA}=$ ophthalmic artery; $\mathrm{CRA}=$ central retina artery; TAMn $=$ time average mean velocity.

Table 2 Effect of dorzolamide on retrobulbar blood flow

\begin{tabular}{|c|c|c|c|c|c|c|c|}
\hline & & $P S V$ & $E D V$ & TAMn & PI & $R I$ & Flow \\
\hline \multirow[t]{2}{*}{ OA } & Before dorzol. & $30.5 \pm 3.1$ & $2.5 \pm 0.6$ & $5.3 \pm 0.8$ & $3.13 \pm 0.22$ & $0.92 \pm 0.01$ & $12391 \pm 113$ \\
\hline & After dorzol. & $32.3 \pm 1.7$ & $3.0 \pm 0.3$ & $5.4 \pm 0.4$ & $3.06 \pm 0.16$ & $0.91 \pm 0.01$ & $11912 \pm 1220$ \\
\hline \multirow[t]{2}{*}{ CRA } & Before dorzol. & $8.2 \pm 0.9$ & $1.4 \pm 0.2$ & $1.8 \pm 0.2$ & $2.14 \pm 0.13$ & $0.82 \pm 0.03$ & $949 \pm 113$ \\
\hline & After dorzol. & $9.3 \pm 1.1$ & $1.6 \pm 0.2$ & $2.0 \pm 0.2$ & $2.03 \pm 0.13$ & $0.81 \pm 0.02$ & $1247 \pm 121$ \\
\hline \multirow[t]{2}{*}{ SPCA } & Before dorzol. & $8.4 \pm 0.7$ & $1.7 \pm 0.1$ & $1.8 \pm 0.1$ & $1.83 \pm 0.15$ & $0.78 \pm 0.02$ & Not determined \\
\hline & After dorzol. & $10.1 \pm 1.3$ & $2.3 \pm 0.2^{*}$ & $2.3 \pm 0.2$ & $1.63 \pm 0.13^{*}$ & $0.74 \pm 0.02^{*}$ & Not determined \\
\hline \multirow[t]{2}{*}{ LPCA } & Before dorzol. & $13.3 \pm 1.1$ & $2.4 \pm 0.1$ & $2.8 \pm 0.2$ & $1.86 \pm 0.10$ & $0.81 \pm 0.02$ & Not determined \\
\hline & After dorzol. & $17.0 \pm 1.5^{*}$ & $3.3 \pm 0.4^{*}$ & $3.7 \pm 0.3^{*}$ & $1.87 \pm 0.12$ & $0.80 \pm 0.02$ & Not determined \\
\hline
\end{tabular}

$\mathrm{PSV}=$ peak systolic velocity; $\mathrm{EDV}=$ end diastolic velocity $\mathrm{TAMn}=$ time average mean velocity; $\mathrm{PI}=$ pulsatility index $=(\mathrm{PSV}-\mathrm{EDV}) / \mathrm{TAMx} ; \mathrm{RI}=\mathrm{resistive}$ index $=(\mathrm{PSV}-\mathrm{EDV}) / \mathrm{PSV}$.

All velocities are given in $\mathrm{cms}^{-1}$; flow is presented in $\mu \mathrm{lmin}^{-1}$.

Asterisk $\left(^{*}\right)$ denotes statistical significance.

\section{Investigator dependency}

vCDI measurements differed statistically significantly between both CDI operators, while both investigators had the same results in standard velocity measurements ( $n=20$; Table 1).

\section{Correlation with Langham method}

No correlation between the vCDI parameters and the LOBF readings could be identified (OA: Spearman's correlation coefficient $r=-0.15, P=0.19, n=69$; CRA: Spearman's correlation coefficient $r=-0.03, P=0.80, n=69$ ).

\section{Detection of changes in ocular perfusion-induced dorzolamide}

Dorzolamide accelerated blood flow in long and short ciliary arteries as expected ( $n=15$; Table 2; Figure 2 ). The pulsatility and resistance indices remained unchanged in all vessels, except in the SPCA in which the pulsatility and resistance indices were statistically reduced.

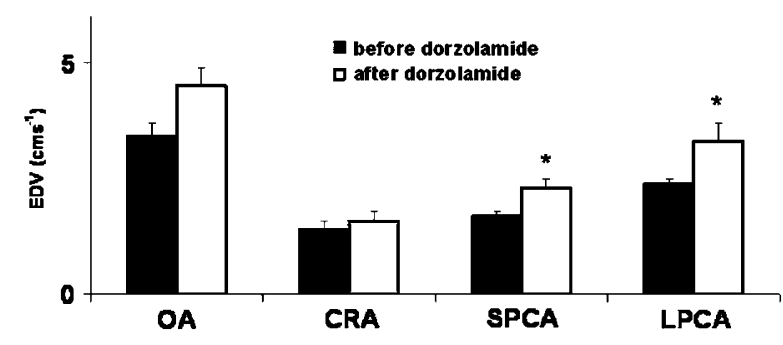

Figure 2 End-diastolic velocity, EDV, before and under therapy with dorzolamide: $\mathrm{OA}=$ ophthalmic artery, $\mathrm{CRA}=$ central retinal artery, SPCA $=$ short posterior ciliary artery, $\mathrm{LPCA}=$ long posterior ciliary artery.

Nevertheless, the flow determined by vCDI in OA and CRA remained unchanged ( $n=15$; Table 2$)$.

\section{Discussion}

The role of disturbed ocular circulation has been subject of intensive investigation for decades. The central problem of this research is methodological. To date there is no method available to determine the most important 
parameter of ocular haemodynamics, the blood flow. If successful, vCDI could contribute to close this gap.

In the present study, the vCDI technique was subjected to a systematic evaluation on a methodological as well as an application level. In summary, the determination of blood volume per time was not reliable due to the variability in vessel diameter recording. To reach an acceptable intraobserver variation, five repeated measurements needed to be averaged. Intraobserver variation of single vCDI measurements is high compared to the interindividual differences. The measures of two independent observers also differ substantially, even if five measures are taken. In contrast to the vCDI-results, reproducibility of flow velocities from the classic CDI method was found to be highly reproducible for both investigators and their results correlated well with one another. This underlines that the negative outcome of the study cannot be attributed to a low experience of the investigators or to a general problem of reliability of CDI measurements. One of the investigators had an over 2-year CDI experience (OZ), the other 6 months (SEV). Experience of the investigators and internal quality control is important because it is known that CDI in general is a subjective measurement. ${ }^{17}$ In our laboratory, we reach acceptable reproducibility of the CDI measurements by maximal standardization of the CDI procedure. $^{18}$

Compared to previous reports, the diameter of the OA as well as that of the CRA appears to be slightly overestimated. ${ }^{10,19}$ In the article from Orge et al, the OA diameter was $2.02 \pm 0.46 \mathrm{~mm}$, and in our study, $2.21 \pm 0.31 \mathrm{~mm}$. In Michelson's work, the diameter was below $2 \mathrm{~mm}$. The true in vivo diameter is not known either for the OA or for the CRA. The dilated ophthalmic vein in crying babies and patients with carotideocavernosus sinus fistula can be well depicted in a B-scan and its diameter measured from this B-scan is $1 \mathrm{~mm}$ or above. Assuming that the measurement of a dilated ophthalmic vein as precise, which is not necessarily true, the inner diameter of the OA, that can never be detected in a B-scan has to be smaller than $1 \mathrm{~mm}$. On the other hand, the course of the OA through the orbit is tortuous. This might contribute to the fact that the normal OA can never be identified in conventional B-scans. The tortuous course requires the CDI operator to visualize the longest possible segment of the OA. Measurement of the diameter can only be carried out in a segment free of visible bends and branches. This was ensured by rotation of the CDI probe. A further problem complicating the measurements of vessel diameter is the alignment of the vessels relative to the ultrasound beam. The spatial distribution of two points can be detected more precisely if the two points are aligned parallel to the direction of the ultrasound beam. In conventional ophthalmologic ultrasound imaging and also in in CDI, the vessels are oriented more or less parallel to the ultrasound beam, resulting in a vertical orientation of the diameter.

A study published previously by our group showed correlations between measures of different methodologies for the assessment of ocular perfusion. ${ }^{16}$ Methods can strengthen each other by these correlations. vCDI measurements did not correlate with the readings from the method of Langham. This can be interpreted as a further indicator of low reliability of vCDI-measures, although one needs to take into account that the method of Langham assesses the total pulsatile blood flow of the choroid, while it is intended by vCDI to measure local blood flow in selected vessels. This methodological difference might also contribute to the lack of correlations between the methods. Despite these considerations, when taking all data about vCDI from this study together, it appears most likely that the missing correlation can be attributed to the insufficient reliability of vCDI.

Observation of changes in ocular perfusion after dorzolamide treatment served as a positive control in the present study. It is reported that systemic carbonic anhydrase (CA) inhibitors may dilate ocular blood vessels. ${ }^{20}$ In addition, several reports address the haemodynamic properties of the topical CA inhibitor compounds. Regarding dorzolamide, positive effects on ocular haemodynamics are reported. ${ }^{13-15}$ With the results from the present study, we were able to reproduce the previously described accelerating effects of dorzolamide on ocular blood flow and reduction of resistance in the SPCA, but, in contrast, could not determine a change in vCDI readings. This is a further indicator that vCDI in its present form is not applicable for use as a clinical diagnostic tool or for scientific purposes.

Although vCDI is not reliable enough for further applications at present, the general idea behind the vCDI concept remains highly attractive not only because of its noninvasive character. Future research should be directed to improving imaging techniques for the orbital, funduscopically invisible vessels in order to get more reliable information about the vessels' diameter. It is unclear whether ultrasound imaging techniques can be optimized to reach this goal. More intensive collaboration between developers of high-end ultrasound set-ups and ophthalmologists are required to optimize modern hardware and software for the special requirements of orbital ultrasound imaging.

\section{Acknowledgements}

We acknowledge Birgit Bischoff and Fatima Icagic gratefully for their excellent technical support of the study. 


\section{References}

1 Schumann J, Orgul S, Gugleta K, Dubler B, Flammer J. Interocular difference in progression of glaucoma correlates with interocular differences in retrobulbar circulation. $A m$ Ophthalmol 2000; 129: 728-733.

2 Boehm AG, Pillunat LE, Koeller U, Katz B, Schicketanz C, Klemm $\mathrm{M}$ et al. Regional distribution of optic nerve head blood flow. Graefes Arch Clin Exp Ophthalmol 1999; 237: 484-488.

3 Langham ME, To'Mey KF. A clinical procedure for the measurements of the ocular pulse-pressure relationship, the ophthalmic arterial pressure. Exp Eye Res 1978; 27: 17-25.

4 Schmidt KG, Ruckmann AV, Mittag TW, Hessemer V, Pillunat LE. Reduced ocular pulse amplitude in low tension glaucoma is independent of vasospasm. Eye 1997; 11: 485-488.

5 Michelson G, Schmauss B, Langhans MJ, Harazny J, Groh MJ. Principle, validity, reliability of scanning laser Doppler flowmetry. J Glaucoma 1996; 5: 99-105.

6 Richard GW. Differentiation of retinal circulation times by videoangiography. Ophthalmologica 1985; 191: 161-163.

7 van Stokkum IH, Lambrou GN, van den Berg TJ. Hemodynamic parameter estimation from ocular fluorescein angiograms. Graefes Arch Clin Exp Ophthalmol 1995; 233: 123-130.

8 Lieb WE, Flaharty PM, Sergott RC, Medlock RD, Brown GC, Bosley $\mathrm{T}$ et al. Color Doppler imaging provides accurate assessment of orbital blood flow in occlusive carotid artery disease. Ophthalmology 1991; 98: 548-552.

9 De Simone R, Glombitza G, Vahl CF, Meinzer HP, Hagl S. Three-dimensional color Doppler flow reconstruction, its clinical applications. Echocardiography 2000; 17: 765-771.

10 Orge F, Harris A, Kagemann L, Kopecky K, Sheet CW, Rechtman $\mathrm{E}$ et al. The first technique for non-invasive measurements of volumetric ophthalmic artery blood flow in humans. Br J Ophthalmol 2002; 86: 1216-1219.
11 Nemeth J, Harkanyi Z, Humml F. Non-invasive volumetric blood flow measurement in the orbit. Br J Ophthalmol 2003; 87: 927-928.

12 Hedges TR. Ophthalmic artery blood flow in humans. $\mathrm{Br} J$ Ophthalmol 2002; 86: 1197.

13 Harris A, Jonescu-Cuypers CP, Kagemann L, Nowacki EA, Garzozi H, Cole C et al. Effect of dorzolamide timolol combination versus timolol $0.5 \%$ on ocular bloodflow in patients with primary open-angle glaucoma. Am J Ophthalmol 2001; 132: 490-495.

14 Avunduk AM, Sari A, Akyol N, Öztürk O, Kapicioglu Z, Erdöl $\mathrm{H}$ et al. The one-month effects of topical betaxolol, dorzolamide, apraclonidine on ocular blood flow velocities in patients with newly diagnosed primary open-angle glaucoma. Ophthalmologica 2001; 215: 361-365.

15 Schmidt KG, von Ruckmann A, Pillunat LE. Topical carbonic anhydrase inhibition increases ocular pulse amplitude in high tension primary open angle glaucoma. Br J Ophthalmol 1998; 82: 758-762.

16 Zeitz O, Matthiessen E, RichardG, Klemm M. Estimation of choroid perfusion by colour Doppler imaging vs other methods. Ultrasound Med Biol 2002; 28: 1023.

17 Polska E, Polak K, Luksch A, Fuchsjager-Mayrl G, Petternel V, Findl $\mathrm{O}$ et al. Twelve hour reproducibility of choroidal blood flow parameters in healthy subjects. $\mathrm{Br} \mathrm{J}$ Ophthalmol 2004; 88: 533-537.

18 Matthiessen ET, Zeitz O, Richard G, Klemm M. Reproducibility of blood flow velocity measurements using colour decoded Doppler imaging. Eye 2004; 18: 400-405.

19 Michelson G, Schuierer G. Absolute blood flow in the ophthalmic artery. Fortschr Ophthalmol 1991; 88: 687-689.

20 Reber F, Gersch U, Funk RW. Blockers of carbonic anhydrase can cause increase of retinal capillary diameter, decrease of extracellular, increase of intracellular $\mathrm{pH}$ in rat retinal organ culture. Graefes Arch Clin Exp Ophthalmol 2003; 241: $140-148$ 